\title{
The Influence of Thermal Dependency of Pad Stiffness on the Measurement of Rail Axial Load based on a Vibration Method
}

\author{
H. Takahashi ${ }^{1}$, K. Abe ${ }^{2}$, F. Urakawa ${ }^{3}$ and K. Koro ${ }^{1}$ \\ ${ }^{1}$ Graduate School of Science and Technology, Niigata University, Japan \\ ${ }^{2}$ Department of Civil Engineering \& Architecture, Niigata University, Japan \\ ${ }^{3}$ Railway Technical Research Institute, Kokubunji, Japan
}

\begin{abstract}
A measuring method of rail axial load based on vibration modes is discussed. In particular, the influence of the thermal dependency of rubber pads on the natural frequency of the pinned-pinned mode is considered. To this end, in the numerical track model a rotational spring depending on the temperature is attached at each rail support. The identification of the temporal equivalent rotational stiffness reflecting the rail pad and fastener is then attempted. Numerical results show that the identification using the ratio of bending moment to rotation angle of rail measured around the rail support has the best performance. In this case, the measurement with an error of $0.1 \%$ is required to assure the identification accuracy of $\pm 50 \mathrm{kN}$.
\end{abstract}

Keywords: continuous welded rail, rail axial load, pinned-pinned mode, resonant frequency, pad stiffness, thermal dependency, identification.

\section{Introduction}

Employment of continuous welded rails (CWR) contributes to the improvement of the passenger comfort and the reduction of vibration and noise. In spite of this advantage, the CWR has a serious issue that the track may suffer buckling or breaks due to the thermal stress. Regarding the CWR track, elastic longitudinal displacement of rails is restrained except both ends of rails. Therefore, thermal stress of the rails inevitably occurs in the longitudinal direction according to variations in temperature. For example, excessive compressive stress by the intense heat in the summer season often causes buckling of track, and the excessive tensile stress by the bitter cold weather in the winter sometimes induces breakage of rails. Therefore, it is very important to 
monitor the axial load of rails in the context of the maintenance of railway.

In the past few decades a number of measuring methods have been proposed. Among them, a method based on displacement measurement will be the most practical and popular one. In order to evaluate the axial load, the longitudinal strain of a rail is calculated from the relative displacement between two observation points on the track. Although this method gives an average value within a range of rail, local strain on the rail surface obtained with a strain gauge or other instruments [1] is also available. While these are methods for evaluating the strain based on the macroscopic deformation of the rail, indirective approaches utilizing physical phenomena such as the magnetoelastic effect [2] and the ultrasonic properties [3-5] have been proposed as other candidates. A drawback common to the abovementioned methods is the necessity of initial state. For example, since the rail stress consists of the elastic and the thermal stresses, the increment of strain and temperature since the rail installation is needed for the evaluation of the absolute stress. Therefore, these methods are not applicable to existing tracks. Application of the X-ray stress measurement technique is also attempted [6]. However, this approach gives microscopic stresses at a point in the vicinity of the rail surface. Since, in general, residual stresses distribute inhomogeneously inside the rail, it is difficult to evaluate the axial load which is given as the resultant of normal stress on a section of rail.

A method which can obtain the absolute longitudinal stress and has already been applied to onsite measurement is the lift method [7-9]. This procedure consists of two steps, i.e. the rail releasement from fasteners over the distance of $30 \mathrm{~m}$ and the rail lifting. A principle that the flexural rigidity of rail depends on the axial load enables us to measure it without the initial state. However, this technique is available only for tensional stress and takes rather a long time due to unfastening over the long distance.

The vibration method is applicable not only to tensional but to compressional absolute stress [10-12]. In this method, utilizing a phenomenon that the vibration modes depend on the axial load, its value is estimated from the resonant frequencies. In spite of this simplicity, since the influence of rail support conditions is innegligible and these are unknowns, it is very difficult to realize.

A similar technique to the vibration method has been proposed $[13,14]$. In this method a rail span released from several fasteners is forced at a constant frequency and the wavenumber of the lateral bending mode is observed. The axial load is estimated using its linear relation to the wavenumber change from the stress-free state. Since the guided wave modes are independent of the rail supports, this method is not affected by any boundary conditions. However, in order to ensure a required quality, very high accuracy is needed for the evaluation of parameters such as the wavenumber of stress-free mode.

As mentioned above, both the vibration and the wavenumber methods have yet problems should be overcome. The authors and collaborators have investigated the feasibility of the former approach from various points of view. In [15] the dispersion analysis of an axially compressed infinite rail which is discretely supported by sleepers was achieved using a three-dimensional Timoshenko beam model. Based on this re- 
sult, the influence of the stiffness of pads and fasteners on the relationship between the natural frequencies of standing wave modes and the axial load was discussed. It was found that the pinned-pinned mode which has nodes at each sleeper position is sensitive to the axial load while rather insensitive to the rail supports, therefore is suitable for the present purpose. The axial stress-natural frequency relation of an infinite periodic track having a constant curvature was obtained by dispersion analysis [16]. The influence of randomness in sleeper spacing on the resonant frequency was discussed in [17], and the importance of this stochasticity was shown. In [18] applicability of the method to tracks having a switch was studied. To this end, an impedance matrix representing a semi-infinite axially stressed CWR has been derived. Effect of mechanical nonlinearlity in rail supports was examined in [19]. It was found that, under this condition, the amplitude of harmonic load does not affect the sensitivity of the resonant frequency to the axial stress but the frequency itself. While in these works numerical experiments were employed, a field experiment was conducted [20] and a linear relationship between the axial load and the resonant frequency was observed.

These results support the capability of the vibration method. However, as indicated by many researchers, it is necessary for its utilization to remove the influence of support conditions. Besides, experimental results [20] claimed that the sensitivity of the resonant frequency to the axial load of a numerical model is lower than that of the real track. This result implies that the conventional numerical model is insufficient to capture the dynamic behavior of tracks.

Since in the above mentioned site experiment the rail axial stress was caused by the thermal change, the stiffness of rail pads made of rubber might be reduced during the thermal loading. This effect induces the reduction in the rotational stiffness of rail pad and then the decrease in the resonant frequency of the track. Therefore, the larger the thermal stress, the larger the frequency reduction. As a result of this, the thermal dependency of pad stiffness will lead to the increase of the apparent sensitivity of resonant frequency to the rail axial load.

In order to verify the above hypothesis, in this paper a numerical track model consisting of a rail, sleepers and pads is considered. The rotational stiffness at a rail fastener is represented by a rotational spring attached at the rail support. The dependence of the spring constant on the temperature is taken into account in the numerical model. Through numerical analyses, the influence of the thermal dependency of the rotational stiffness on the relationship between the rail axial load and the resonant frequency is investigated.

This modeling implies that not only the resonant frequency but also the pad stiffness and its thermal dependency are necessary for the measurement of rail axial load. However, in general, these properties will have some randomness and may suffer time related deterioration. Therefore, it will not be a practical strategy to reflect these aspects in the numerical model a priori. To cope with this difficulty, in this paper an identification of the temporal equivalent rotational stiffness is attempted based on a numerical model.

In Section 2, influence of the thermal dependency of pad stiffness on the reso- 
nant frequency of a track is discussed based on numerical and experimental results. Section 3 describes the vibration mode analysis which is used in the identification of rotational stiffness of a rail support. Physical quantities used as objective functions for the identification are described in Section 4. In Section 5, the identification by these quantities is attempted through numerical experiments, and error tolerance of measurements required to assure identification accuracy is examined.

\section{Influence of thermal dependency of pad stiffness on dynamic nature of axially stressed rail}

\subsection{Track modeling}

In this study time-domain dynamic response analyses of a finite track model are substituted for the measurement. The track model consists of a rail subjected to an axial load $N$, with sleepers and pads as shown in Figure 1. The ballast is represented by Viogt units. In general, the rail is restrained somewhat from the rotation due to the existence of rail pad and fastener at each sleeper. To take into account this effect, an equivalent rotational spring $k$ is attached at every rail support. Although the ballast has also the rotational stiffness, the temperature dependency is existing only in the rail pad. As shown in Figure 3, this effect can be captured by the simple model illustrated in Figure 1. Therefore, only the rotational stiffness of rail pad is considered.

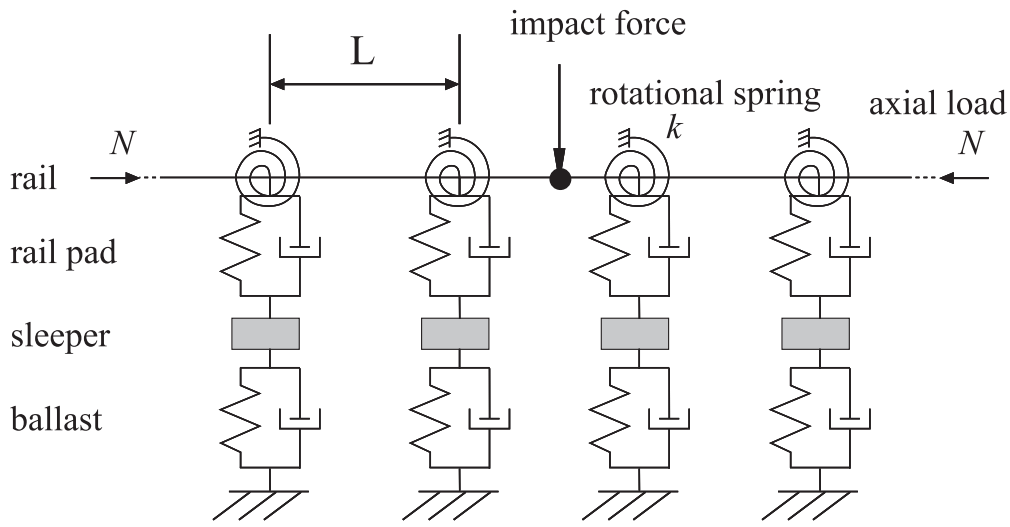

Figure 1: Track model for time-domain response analysis

It is assumed that in the measurement the track vibration is excited with an impulse hummer. To simulate this, a unit impact force is applied at a midspan for $10 \times 10^{-4}$ second as shown in Figure 2, with time increment of $5 \times 10^{-5}$ second. In accordance with the experiments performed by Aikawa, et al. [20], the Japanese $50 \mathrm{kgN}$ rail is 


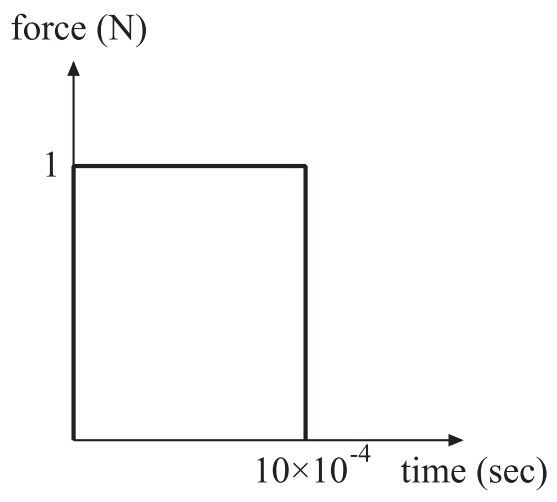

Figure 2: Unit impact force applied at a midspan

assumed. The rail is modeled as a Timoshenko beam and discretized by 12 elements for each sleeper span. The track consists of 360 sleeper bays. Parameters in the track model are summarized in Table 1. In this table parameters of rail and sleeper are set based on the experiment. Since the mechanical conditions of the rail pad and ballast are insignificant in the pinned-pinned mode, standard values are assumed. Since, in

\begin{tabular}{|c|c|c|}
\hline & mass density $\left(\mathrm{kg} / \mathrm{m}^{3}\right)$ & 7880 \\
& cross-sectional area $\left(\mathrm{m}^{2}\right)$ & $64.05 \times 10^{-4}$ \\
& Young's modulus $(\mathrm{GPa})$ & 206 \\
& Poisson's ratio & 0.33 \\
& geometrical moment of inertia $\left(\mathrm{m}^{4}\right)$ & $1960 \times 10^{-8}$ \\
& shear factor & 0.394 \\
\hline sleeper & mass $(\mathrm{kg})$ & 80 \\
& spacing $(\mathrm{m})$ & 0.7374 \\
\hline rail pad & spring constant $(\mathrm{MN} / \mathrm{m})$ & 120 \\
& damping coefficient $(\mathrm{kN} \cdot \mathrm{s} / \mathrm{m})$ & 100 \\
\hline ballast & spring constant $(\mathrm{MN} / \mathrm{m})$ & 30 \\
& damping coefficient $(\mathrm{kN} \cdot \mathrm{s} / \mathrm{m})$ & 50 \\
\hline
\end{tabular}

Table 1: Parameters in track model

general, distribution of the rail axial load is not uniform, it is desirable to investigate the influence of the stress variation on the present method. However, in this study we focus only on the effect of rotational stiffness of rail supports. Therefore, the longitudinal variation of the axial load is not modeled. In the following, vertical loading and deflection are considered. Based on an experimental result [21], in this paper the 
temperature dependency of stiffness is expressed by the following relation :

$$
k=\frac{k_{0}\left(T_{0}+42.12\right)}{T+42.12}
$$

where $k_{0}$ is the rotational stiffness corresponding to the temperature $T_{0}$. These values are set as $k_{0}=5.21 \mathrm{MN} \cdot \mathrm{m} / \mathrm{rad}$ and $T_{0}=20^{\circ} \mathrm{C}$. In order to reproduce the sensitivity of the resonant frequency to the axial load in [20], based on a pre-analysis, the rotational stiffness at each rail fastener is set rather higher. Notice that, in a real track the apparent pad stiffness will be increased due to the damping force under high frequency vibration.

The rail axial load is introduced as the thermal stress. Hence, both of the axial load and the rotational stiffness are given as functions of the temperature.

\subsection{Numerical Results}

As mentioned in [17], the pinned-pinned mode which has nodes at each sleeper support is one of the most suitable modes for assuming the axial load distribution among the standing wave modes of track. In the numerical experiments the resonant frequency corresponding to the pinned-pinned mode is identified from the Fourier spectrum of rail acceleration obtained by the time-domain response analysis. The observation point of the rail deflection is located at the loading point.

The relationship between the pinned-pinned resonant frequency and the rail axial load is shown in Figure 3. The numerical result for a track model with pad stiffness independent of the temperature is also plotted. Solid lines are theoretical relationship between the axial load and the resonant frequency obtained for different pad stiffness $k$. The slopes of these lines are identical, and determined by mode analysis which will be mentioned in Section 3. The dashed line is linear regression of the experiment. "Relative resonant frequency" in the figure stands for the relative value from the resonant frequency of uncompressed rail, i.e. $N=0$. The numerical result with the thermal dependency plotted by filled circles is obtained for the pad model of Equation (1). Since in this model the rotational pad stiffness is given by a function of the temperature, its value decreases with increasing the thermal stress. From the figure, it can be seen that the numerical result for the temperature dependent model is in good agreement with the experiment of [20], while the temperature independent model has an obvious discrepancy. Consequently, the consideration of the thermal dependency of pad stiffness is essential in the context of the present measuring method.

\section{Vibration mode analysis}

From the above comparison between the numerical analysis and the site experiment, it was found that the consideration of the thermal dependency of rail pads is essential to simulate the present phenomenon. However, this modeling implies that not only the 


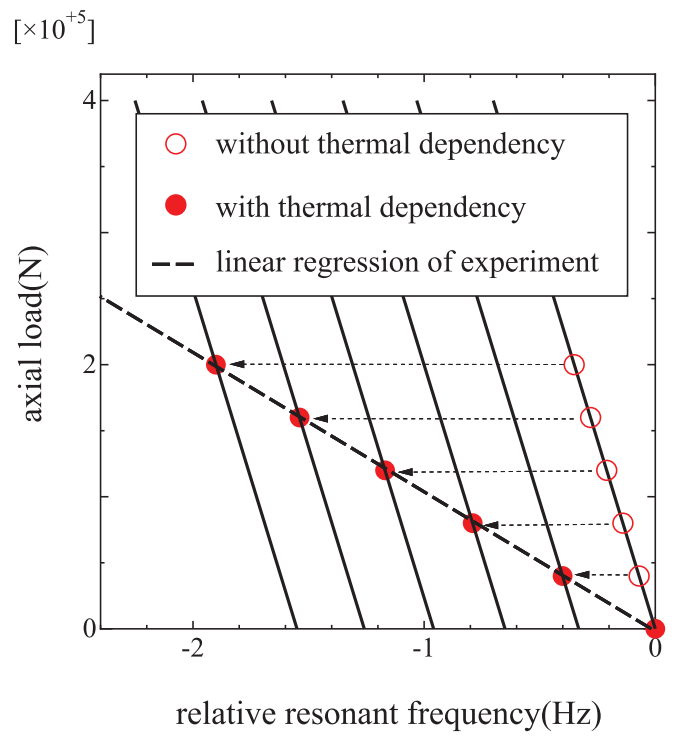

Figure 3: Influence of thermal dependency of rotational stiffness on relation between axial load and relative resonant frequency

resonant frequency but also the pad stiffness and its thermal dependency are necessary for the measurement of the rail axial load. Meanwhile, the rotational stiffness of real track is affected not only by the pads but also by the rail fasteners. Furthermore, since the material parameters of rubber pad may have randomness, it is necessary to know the thermal dependency of pads in each track. However, it is not practical to introduce these effects into a track model used for the axial stress evaluation. From the numerical result shown in Section 2.2, it can be understood that, once the resonant frequency $f_{0}$ at $N=0$ is obtained for the present rotational stiffness, the axial load can be evaluated by its theoretical sensitivity to the frequency. Since this resonant frequency $f_{0}$ depends on the rotational stiffness, the present issue is reduced to the identification of the equivalent rotational stiffness. Therefore, we set the equivalent rotational stiffness that represents all effects as unknown quantities. In order to identify the temporal rotational stiffness based on the numerical model, we consider the eigenvalue problem of an infinite track. The identification of the unknown stiffness will be achieved based on the eigenmode. In this section the outline of the mode analysis is described.

\subsection{Modeling of infinite track}

In general, a CWR track can be represented as a periodic structure characterized by the sleeper spacing $L$. A dynamic problem of this infinite system can then be reduced 


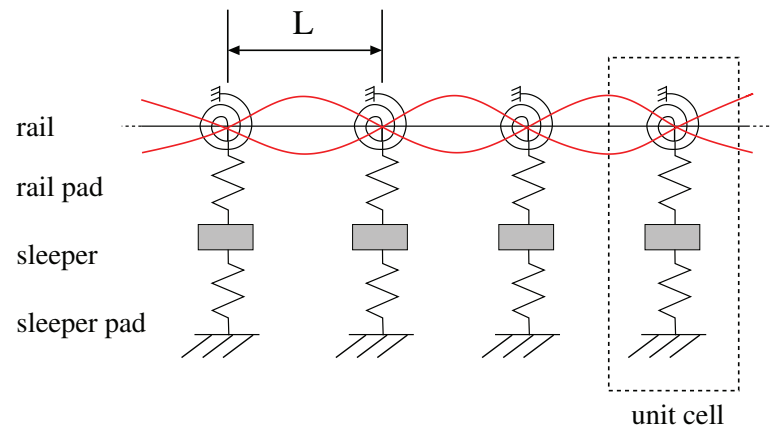

Figure 4: Unit cell of track model and pinned-pinned mode

to that of a substructure called unit cell as shown in Figure 4. In this study a unit cell consisting of a rail, a sleeper and springs representing pad and ballast is taken into account. In order to reproduce the moment that acts at the rail pad, each rail support is modeled by a rotational spring as in the time-domain analysis.

\subsection{Eigenvalue problem}

The equation of motion of a unit cell is given by

$$
[\overline{\mathbf{W}}]^{T}\left[\mathbf{K}-N \mathbf{C}-\omega^{2} \mathbf{M}\right]\{\mathbf{U}\}=[\overline{\mathbf{W}}]^{T}\{\mathbf{F}\}
$$

where $\{\mathbf{W}\}$ is a virtual displacement vector, $\{\mathbf{U}\}$ and $\{\mathbf{F}\}$ are nodal displacement and force vectors. $\left(^{-}\right)$and ()$^{T}$ stand for conjugate and transpose. $[\mathbf{K}]$ and $[\mathbf{M}]$ are the stiffness and mass matrices, respectively. $\omega$ is the circular frequency. $[\mathbf{C}]$ is a matrix concerning the axial load $N$.

By virtue of the Floquet's principle [22], a steady state solution satisfies the following relation

$$
\mathbf{u}_{L}=\mathbf{u}_{0} e^{-i \kappa L}, \quad \mathbf{f}_{L}=-\mathbf{f}_{0} e^{-i \kappa L}
$$

where $\mathbf{u}_{0}$ and $\mathbf{u}_{L}$ are nodal displacements at both ends of $x=0$ and $x=L . \mathbf{f}_{0}$ and $\mathbf{f}_{L}$ are nodal internal force vectors at these points. $\kappa$ is a propagation constant.

Equations (2) and (3) lead to an eigenvalue problem as

$$
\left[\mathbf{K}^{\prime}-N \mathbf{C}^{\prime}\right]\left\{\mathbf{U}^{\prime}\right\}=\omega^{2}\left[\mathbf{M}^{\prime}\right]\left\{\mathbf{U}^{\prime}\right\}
$$

where ()$^{\prime}$ denotes that the matrices and displacement vector have been degenerated due to the elimination of $\mathbf{u}_{L}$ and $\mathbf{f}_{L}$ using the relations of Equation (3). Notice that components of the Hermitian matrices $\left[\mathbf{K}^{\prime}\right],\left[\mathbf{C}^{\prime}\right]$ and $\left[\mathbf{M}^{\prime}\right]$ are given by functions of the propagation constant $\kappa$. Therefore, the eigenvalue problem of Equation (4) gives the dispersion structures, i.e. the relation between $\kappa$ and $\omega$ for wave modes propagating in the track. The pinned-pinned mode is given by a standing wave mode at $\kappa=\pi / L$. 


\section{Identification of rotational stiffness}

In order to identify the equivalent rotational stiffness, we consider physical quantities that depend on the rotational stiffness.

\subsection{Physical quantity given by ratio of bending moment to rota- tion angle}

A simplified model of rail and rail fastner is shown schematically in Figure 5. As-

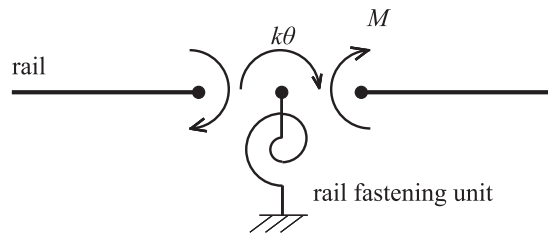

Figure 5: Schematic illustration of rail and fastener

suming that at both points on the left and right sides of a rail fastener, the dynamic response is in the nearly antisymmetric state for the pinned-pinned resonance, the equilibrium condition of moment at the rail fastening unit is thus given by

$$
2 M+k \theta=0
$$

where $M$ is the bending moment acting on the rail cross-section at both sides of the rail fastener, and $\theta$ is the angle of rotation at the rail support. In principle, if the bending moment $M$ is measured by a strain gauge, the rotational stiffness $k$ can be evaluated from Equation (5). However, in an actual track with a rail fastener, it is impossible to attach a strain gauge to a rail fastening position. Therefore, the equilibrium condition given by Equation (5) is unavailable to identify $k$. To cope with this restriction, in this study the bending moment at a different position is employed as the measurement data. Notice that in this case the simple relation of Equation (5) is no longer applicable. Nevertheless, the bending moment will depend on the stiffness $k$. Therefore, the following physical quantity $m_{1}$ depending on $k$ can be used as a target function for the identification,

$$
m_{1}=\frac{M(x)}{\theta}
$$

where $M(x)$ is the bending moment at a certain position $x$.

The rotation angle can be obtained by numerical differentiation of vertical accelerations observed at two neighboring points of the rail support. In this case its accuracy is depends on the measurement accuracy of the accelerometer as $a(x)$ in Equation (7). 


\subsection{Physical quantity given by relative acceleration ratio}

The rail deflection of the pinned-pinned mode will depend on the rotational stiffness $k$ as illustrated in Figure 6. Therefore, as another target function $m_{2}$, we consider a

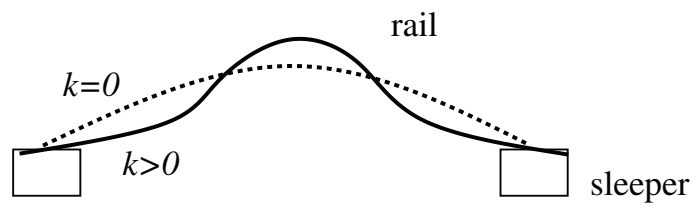

Figure 6: Influence of rotational stiffness on mode shape

representative value of the mode shape that depends on the rotational stiffness. That is, the ratio of the vertical acceleration of the rail at the midspan to that at other position is employed as $m_{2}$. In the actual measurement, the vertical acceleration at the rail support excited by the impact loading will affect the vertical acceleration component. Therefore, in order to elliminate this influence, relative acceleration to the one at the rail fastening position are used as

$$
m_{2}=\frac{\left|a_{m i d}-a_{f}\right|}{\left|a(x)-a_{f}\right|}
$$

where, $a(x), a_{f}$ and $a_{m i d}$ are vertical accelerations at a certain position $x$, the rail fastener and the midspan, respectively (Figure 7). $|\cdot|$ stands for the Fourier amplitude corresponding to the pinned-pinned resonance component.

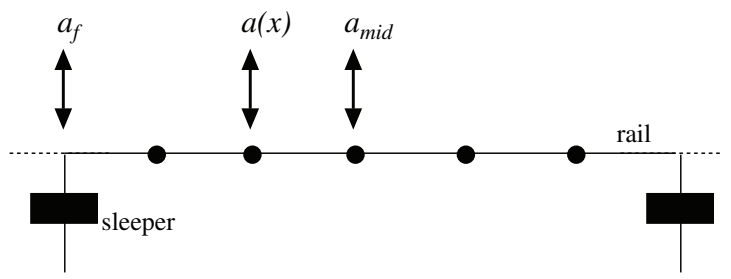

Figure 7: Measuring positions of acceleration

\subsection{Physical quantity given by ratio of bending moment at midspan to relative acceleration}

From numerical experiments, it was found that the absolute value of the bending moment at midspan is about two times larger than the one at the rail fastening position. 
Hence, if the measuring position is located at this portion, the relative error of measurement can be mininized. Therefore, as the third candidate for the physical quantity, the following function $m_{3}$ is also employed

$$
m_{3}=\frac{M_{\text {mid }}}{\left|a(x)-a_{f}\right|}
$$

where $M_{m i d}$ is the bending moment of the rail at the midspan.

\subsection{Identification of $k$ and $N$}

The objective function for each $m_{i}$ is given by

$$
J_{i}=\left(m_{i}-\bar{m}_{i}\right)^{2} \quad(i=1,2,3)
$$

where $\bar{m}_{i}$ is the measurement value corresponding to $m_{i}$. Since $\bar{m}_{i}$ is the quantity relating to the pinned-pinned mode, the acceleration component is to be obtained from the Fourier spectrum of the measurement data. Through various studies, we found that, fortunately, the influence of the axial load $N$ on $m_{i}$ 's is negligible. Since, in this case, the objective function $J_{i}$ depends only on the rotational stiffness $k$, this unknown value can be determined irespective of $N$. Although the rotational stiffness which minimizes the objective function $J_{i}$ can be found by scanning its value in an interval, in this study this unknown is searched by means of the sensitivity analysis.

Once this value is obtained, the resonant frequency of uncompressed rail $f_{0}$ can be determined from the relationship between $k$ and $f_{0}$ as shown in Figure 8. It enables us to attain the relationship between the resonant frequency $f$ and the axial load $N$. The axial load $N$ can then be obtained from $f$ as depicted in Figure 9. Notice that, the slope of the $f-N$ relation can be obtained a priori from the theoretical dependency of the natural frequency of the pinned-pinned mode on the axial load.

This procedure is summarized as follows :

a) Measure $f$ and $m_{i} . m_{i}$ is calculated based on the resonance mode obtained from Fourier amplitude.

b) Identify $k$, so that it can minimize the objective function $J_{i}$. It is achieved through the eigenmode analysis.

c) Evaluate $f_{0}$ from $k$ (Figure 7). $f_{0}$ corresponding to the identified $k$ is obtained by the eigenvalue analysis.

d) Evaluate $N$ from the $f-N$ relation (Figure 8). The slope of the $f-N$ line is obtained by the eigenmode analysis. The resonant frequency for uncompressed rail $f_{0}$ has been determined in $\mathbf{c}$ ), while $f$ has been obtained in a). 


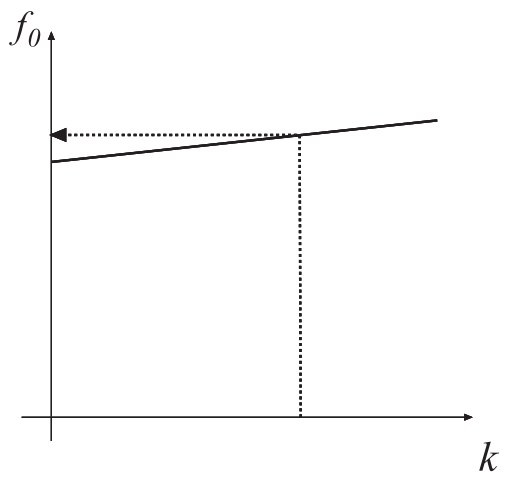

Figure 8: Evaluation of resonant frequency $\left(f_{0}\right)$ based on the value of rotational stiffness of rail pad $(k)$

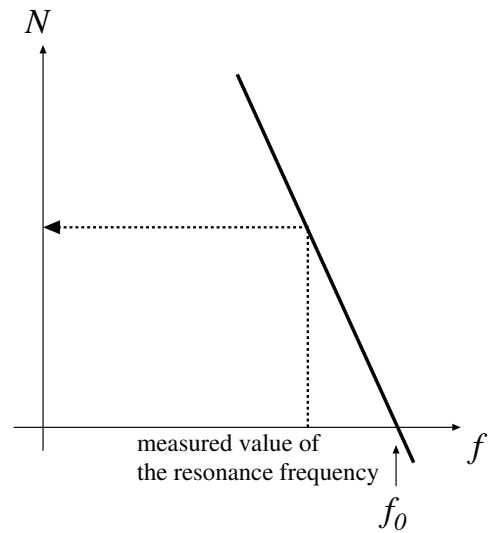

Figure 9: Evaluation of axial load $(N)$ based on the measurement value of resonant frequency $(f)$ using the slope of the $f-N$ relation, which can be obtained a priori from the theoretical dependency of the natural frequency of the pinned-pinned mode on the axial load

\section{Case study}

\subsection{Identification of $k$ and $N$ with $J_{1}$}

The influences of measurement position $x$ of the bending moment $M(x)$ (strain) on the identification accuracy of $k$ and $N$ are shown in Figure 10 for the objective function $J_{1}$ given by $m_{1}$. The initial value of $k$ in the identification calculation is set to 


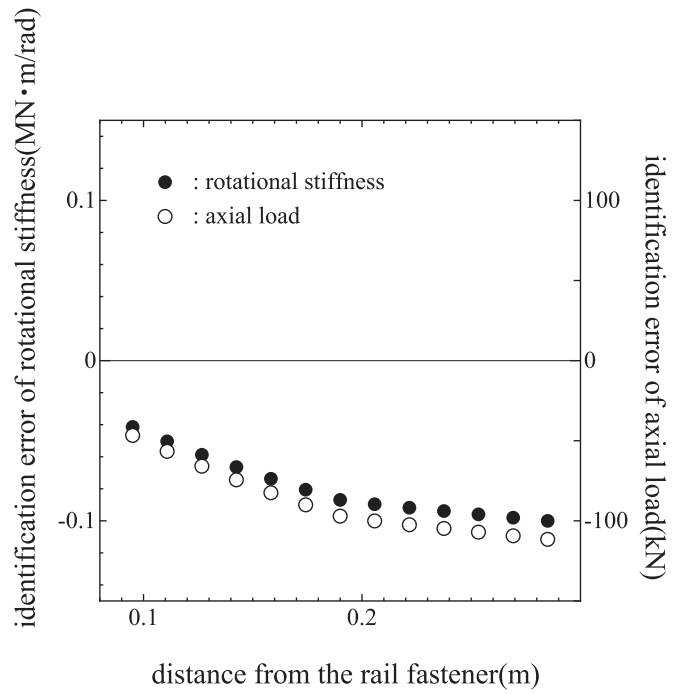

Figure 10: Influence of measurement position of bending moment $M(x)$ on identification error of rotational stiffness $(k)$ and axial load $(N)$. Unknowns are identified with $J_{1}$

4.85(MN.m/rad), and the correct value is $k=5.21(\mathrm{MN} \cdot \mathrm{m} / \mathrm{rad})$, with the axial load of $N=10 \times 10^{4}(\mathrm{~N})$. The sleeper spacing is set to $0.57 \mathrm{~m}$. Other track conditions are identical to those in Section 2.

The measurement position nearest to the fastener $(0.095 \mathrm{~m})$ provides the best accuracy for the identification. Even for the true value of $m_{1}$, the identification of $k$ has some error. As mentioned above, in general, it is expected that the increase of the rotational stiffness $k$ will lead to the decrease of the rotation angle and the amplification of the bending moment at the fastener. Whereas the opposite result was obtained from the pinned-pinned mode analysis, i.e. the higher the stiffness, the larger the rotation angle. Although the cause of this tendency is not clear, the time-domain track vibration analysis which was performed as the simulation of measurement process showed similar results to the mode analysis.

\subsection{Identification of $k$ and $N$ with $J_{2}$}

The influences of the measurement position of vertical acceleration on the identification accuracy of $k$ and $N$ are shown in Figure 11 for the objective function $J_{2}$ given by $m_{2}$. The initial and true values of $k$ and axial load are the same as for $J_{1}$.

The highest accuracy of the identification was obtained for the measurement at $0.111 \mathrm{~m}$ from the fastener. Notice that rather large error is observed at around $0.06 \mathrm{~m}$ 


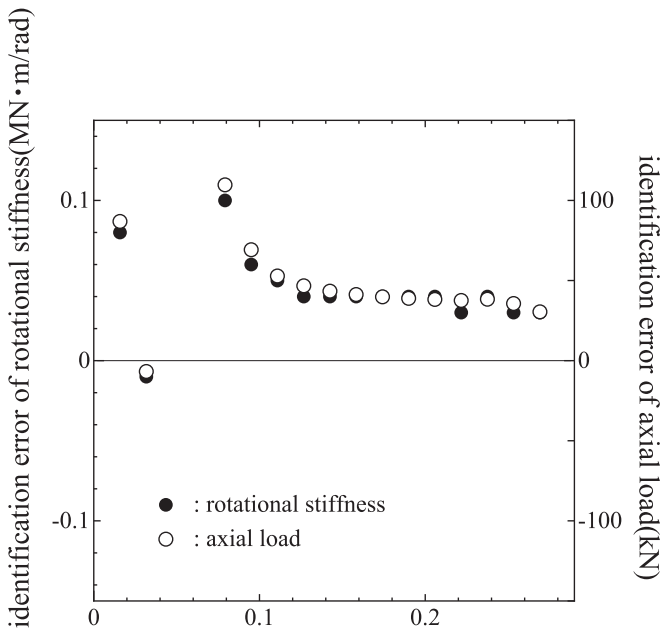

distance from the rail fastener(m)

Figure 11: Influence of measurement position of vertical acceleration $a(x)$ on identification error of rotational stiffness $(k)$ and axial load $(N)$. Unknowns are identified with $J_{2}$

from the center of the rail support. This deterioration will have relation to the fact that rather large bending stiffness due to the existence of the rail fastener is considered in beam elements adjacent to the rail fastening point.

\subsection{Identification of $k$ and $N$ with $J_{3}$}

The influences of the measurement position of vertical acceleration on the identification accuracy of $k$ and $N$ are shown in Figure 12 for the objective function $J_{3}$ given by $m_{3}$. The initial and true values of $k$ and axial load are the same as for $J_{1}$.

It is found that the identification error is independent of the measurement position of vertical acceleration. However, the largest error is obtained for the present identification in the three cases.

\subsection{Evaluation error of axial force caused by measurement error of $m_{i}^{\prime} \mathbf{s}$}

In order to investigate the influence of measurement error of each quantity $m_{i}$ on the estimation of axial force, we calculate the variance of $N$ caused by the change in $m_{i}$. Here, based on the results shown in Sections 5.1, 5.2 and 5.3, the measurement position for each $m_{i}$ is set so that the best performance can be attained. That is, $m_{i}^{\prime} \mathrm{s}$ 


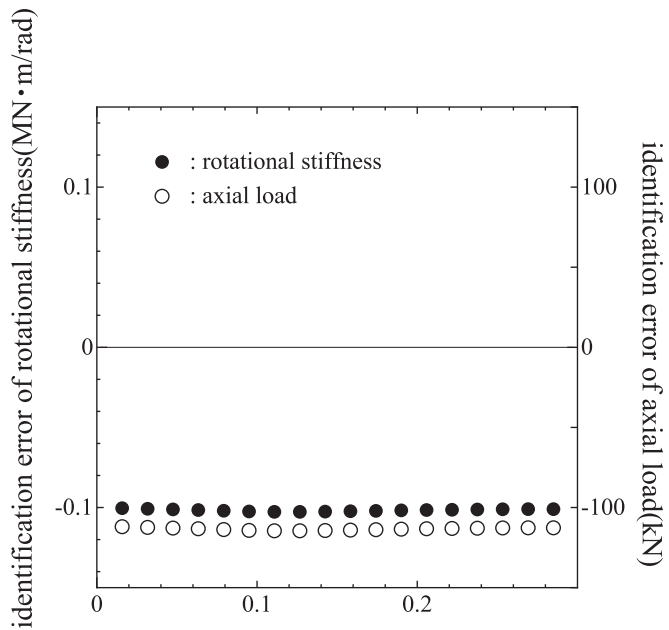

distance from the rail fastener(m)

Figure 12: Influence of measurement position of vertical acceleration $a(x)$ on identification error of rotational stiffness $(k)$ and axial load $(N)$. Unknowns are identified with $J_{3}$

are given by

$$
\begin{gathered}
m_{1}^{\prime}=\frac{M(0.095)}{\theta} \\
m_{2}^{\prime}=\frac{\left|a_{m i d}-a_{f}\right|}{\left|a(0.111)-a_{f}\right|} \\
m_{3}^{\prime}=\frac{M_{\text {mid }}}{\left|a(0.016)-a_{f}\right|}
\end{gathered}
$$

where ( $)^{\prime}$ stands for the best measurement quantity. $M(0.095)$ is the bending moment of the rail at the distance of $0.095 \mathrm{~m}$ from the rail fastener, and $\mathrm{a}(0.111)$ and $\mathrm{a}(0.016)$ are the vertical accelerations at $0.111 \mathrm{~m}$ and $0.016 \mathrm{~m}$ from the rail fastener, respectively.

Variances of $N(\Delta N)$ and $k(\Delta k)$ are given as

$$
\begin{gathered}
\Delta N=\frac{\partial N}{\partial k} \Delta k \\
\Delta k=\frac{\partial k}{\partial m^{\prime}} \Delta m^{\prime}
\end{gathered}
$$

Applying the chain rule to Eqs.(13) and (14), we can obtain the following relation,

$$
\Delta N=\frac{\partial N}{\partial k} \frac{\partial k}{\partial m^{\prime}} \cdot m^{\prime} \cdot \alpha
$$


where $\alpha$ is the relative change of $m^{\prime}$ defined by

$$
\alpha=\frac{\Delta m^{\prime}}{m^{\prime}}
$$

From Equations (15) and (16), we can evaluate $\Delta N$ with respect to a certain relative accuracy $\alpha$ for each measurement quantity. The sensitivity of $N$ to $k$ and that of $k$ to $m_{i}$ are summarized in Table 2 for $m_{i}^{\prime}(i=1,2,3)$. The values of $m_{i}^{\prime}$ shown in this table are obtained by numerical experiment. $\Delta N$ for $\alpha=1 \%$ is also shown in the table. It can be found that the identification with $m_{1}^{\prime}$ has the best performance. However, even in this case, the measurement with error of about $0.1 \%$ or less is required for $m_{1}^{\prime}$ to assure the identification accuracy of $\pm 50 \mathrm{kN}$. This condition seems to be rather severe requirement. In order to satisfy this error tolerance, some measurement technique will be needed.

\begin{tabular}{|c|c|c|c|c|c|}
\hline & $\begin{array}{c}\text { measurement } \\
\text { position }(\mathrm{m})\end{array}$ & $\partial k / \partial m^{\prime}$ & $\begin{array}{c}\partial N / \partial k \\
(\mathrm{rad} / \mathrm{m})\end{array}$ & $m_{i}^{\prime}$ & $\Delta N(\mathrm{kN})$ \\
\hline$m_{1}^{\prime}$ & 0.095 & $-5.564(-)$ & & $6878164(\mathrm{~N} \cdot \mathrm{m} / \mathrm{rad})$ & $-4.3 \times 10^{2}$ \\
$m_{2}^{\prime}$ & 0.111 & $1.081 \times 10^{9}(\mathrm{~N} \cdot \mathrm{m} / \mathrm{rad})$ & 1.12 & $2.0701(-)$ & $2.5 \times 10^{4}$ \\
$m_{3}^{\prime}$ & 0.016 & $-3.228 \times 10^{7}\left(\mathrm{~m} / \mathrm{rad} \cdot \mathrm{s}^{2}\right)$ & & $19.7933\left(\mathrm{~N} \cdot \mathrm{s}^{2}\right)$ & $-7.2 \times 10^{3}$ \\
\hline
\end{tabular}

Table 2: Influence of measurement error of physical quantity $m_{i}$ on evaluation error of axial force

\subsection{Computational example}

In the following, application of the proposed method is demonstrated through a numerical simulation. The track model discussed in this section is considered, i.e. $k=5.21 \mathrm{MN} \cdot \mathrm{m} / \mathrm{rad}$. The rail axial load is evaluated based on $m_{1}^{\prime}$ for the true value of $N=200 \mathrm{MN}$. The unit impact force shown in Figure 2 is applied at the central midspan of the track model. The response in time-domain and its Fourier amplitude are shown in Figures 13 and 14 for the bending moment.

The measurement value $m_{1}^{\prime}=5.84 \mathrm{MN} \cdot \mathrm{m} / \mathrm{rad}$ is given by the ratio of Fourier amplitudes of the bending moment $M_{R}$ to the angle of rotation $\theta_{R}$ obtained for the resonance component. The relation between $m_{1}^{\prime}$ and $k$ obtained by the eigenmode analyses is shown in Figure 15 to this problem. Notice that $k=4.94 \mathrm{MN} \cdot \mathrm{m} / \mathrm{rad}$ is identified based on the objective function $J_{1}$. This value has error of about $5 \%$ for the true value $k=5.21(\mathrm{MN} \cdot \mathrm{m} / \mathrm{rad})$. Once $k$ has been evaluated, $f_{0}=1054.1 \mathrm{~Hz}$ is then obtained from $k-f_{0}$ curve as shown in Figure 16. Finally, the axial load $N$ is evaluated from $f-N$ relation as shown in Figure 17. Here, the measurement value of resonant frequency $f=1054.7 \mathrm{~Hz}$ is substituted into this relation. Although the rotational stiffness $k$ can be identified with rather good accuracy, since the slope of $f-N$ relation is very steep, the small discrepancy in $f_{0}$ leads to large error of about $300 \mathrm{kN}$. It should be bear in 


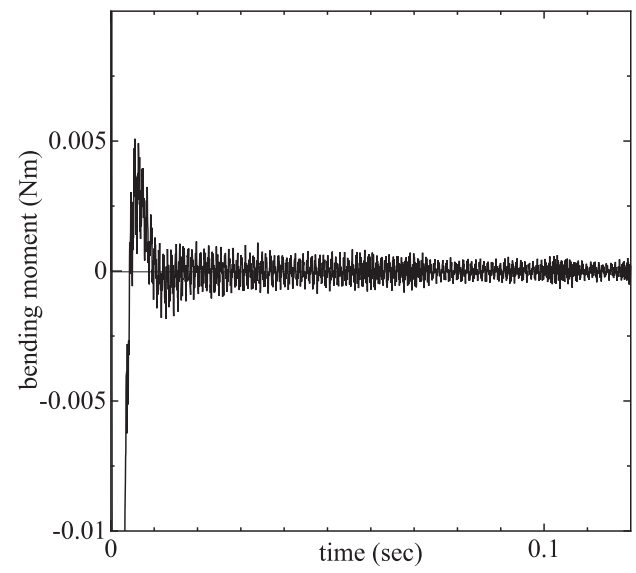

Figure 13: Time-domain response of bending moment of rail at $0.095 \mathrm{~m}$ from the fastener

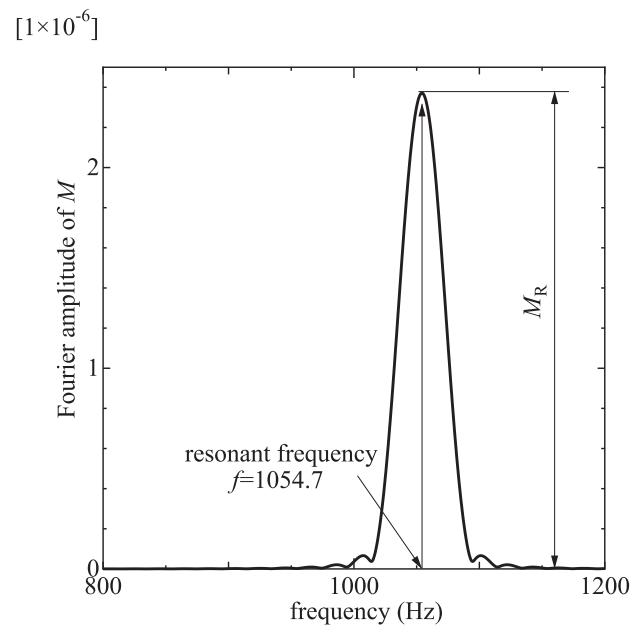

Figure 14: Fourier amplitude of bending moment at $0.095 \mathrm{~m}$ from the fastener.

mind that any disturbances such as signal noise and inhomogeneity in track are not considered in this analysis. Therefore, the site measurement will suffer larger deterioration than this numerical example. As mentioned in Section 5.4, in order to assure the required accuracy, further improvement is needed. As shown in Table 2, the identification with $J_{2}$ or $J_{3}$ is more sensitive to the measurement error than $J_{1}$. Because of this, the estimation error becomes larger than $1 \mathrm{MN}$. 


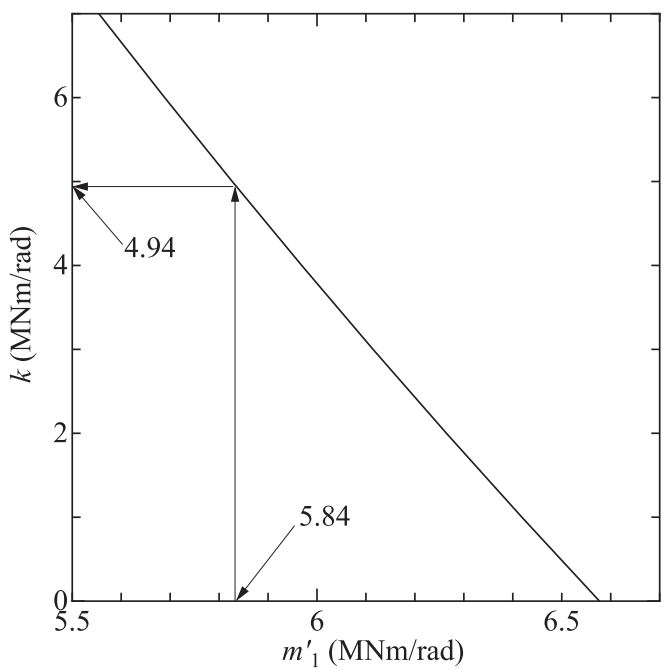

Figure 15: Relation between $m_{1}^{\prime}$ and rotational stiffness $k$

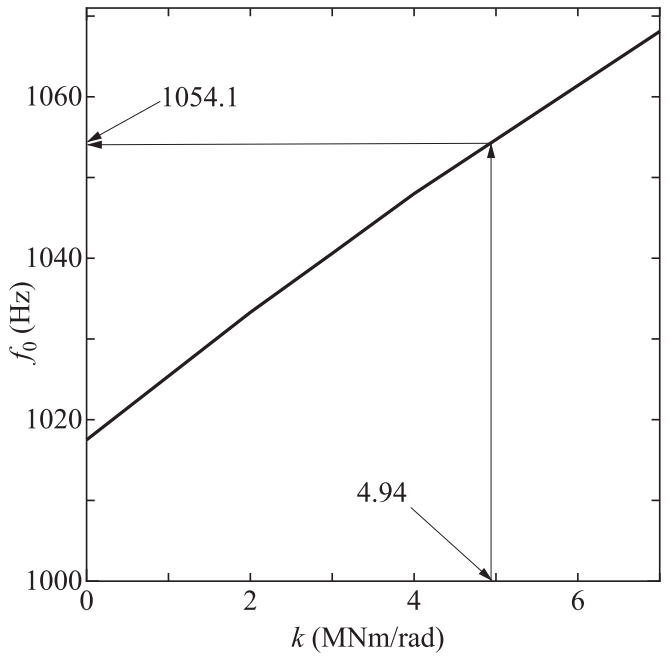

Figure 16: Evaluation of resonant frequency $f_{0}$ of uncompressed rail from rotational stiffness $k$ 


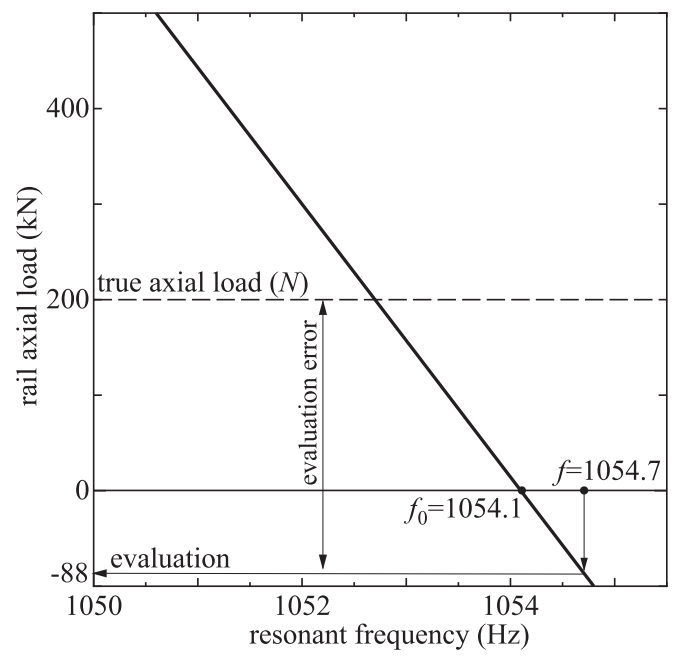

Figure 17: Evaluation of rail axial load $N$ from resonant frequency $f$

\section{Conclusions}

Measurement of rail axial force by the vibration method has been discussed. In order to reproduce the sensitivity of the pinned-pinned resonant frequency to the axial force, which is observed in a real track, the thermal dependency of the rotational stiffness of rail pad was considered. Through numerical experiments, it was found that the influence of the rotational stiffness on the resonant frequency is significant. This result also reveals that the thermal dependency of pad stiffness dominates the apparent relationship between the axial load and the resonant frequency. However, in general, it will not be easy to take into account this effect in a numerical model. To solve this difficulty, in this study the identification of the equivalent rotational stiffness at the rail support was attempted. Once the temporal rotational stiffness is obtained, the axial load can be converted from the resonant frequency using the theoretical relation between them. For this purpose, several quantities reflecting the rotational stiffness were employed as measurement data. Numerical results showed that the quantity defined by the ratio of bending moment to rotation angle of rail measured around the rail support has the best performance. However, even in this case, the measurement with error of about $0.1 \%$ is required to assure the identification accuracy of $\pm 50 \mathrm{kN}$. This condition seems to be rather severe requirement.

Although, in this paper, the estimation of equivalent rotational stiffness is applied only to the nearest sleeper supports, the uncertainty of other supports may be innegligible. Besides, in general, the resonant frequency of the pinned-pinned mode will be affected by the randomness in sleeper spacing [17]. From comparison between the 
site experimental and numerical results in [23], it can also be deduced that the variation of axial load in a range of about 50 sleeper bays or shorter affects the evaluation. Therefore, many obstacles which should be overcome are still left. As a countermeasure, exclusion of track conditions such as the pad stiffness and sleeper spacing by restricting both ends of an unfastened rail region is under examination.

\section{References}

[1] H-J. Yoon, K-Y. Song, J-S. Kim, D-S. Kim, "Longitudinal strain monitoring of rail using a distributed fiber sensor based on Brillouin optical correlation domain analysis", NDT\&E International, 44, 637-644, 2011.

[2] K. Kashiwaya, H. Sakamoto, "Linearization method of output vs. stress in stress measurement using magnetic anisotropy sensor", Journal of JSNDI, 53(2), 9397, 2004 (in Japanese).

[3] J. Szelazek, "Monitoring of thermal stress in continuously welded rails with ultrasonic technique", NDTnet, 3(6), 1998.

[4] H. Toda, H. Tsurumaru, "Axial stress measurement of long welded rails using acoustoelasticity - The first report: Acoustoelastic characteristics of V-reflection longitudinal waves -", Journal of JSNDI, 57(5), 246-252, 2008 (in Japanese).

[5] H. Toda, T. Kodama, Y. Murata, "Axial stress measurement of long welded rails using acoustoelasticity - The second report: Measurement of monthly variation of axial stress in a newly constructed rail using advanced equipment -", Journal of JSNDI, 57(9), 441-447, 2008 (in Japanese).

[6] Y. Kanematsu, M. Matsui, "Fundamental study of simulated rail axial force by X-ray stress measurement method", Proceedings of J-Rail (CD-ROM), 2015 (in Japanese).

[7] J.P.A. van Tonder, "Determining the stress-free rail temperature of continuous welded rails (CWR)", UIC-ERRI Interactive Conference, Paris, 1-6, 1998.

[8] C. Lemon, R.J. Gostling, "The non-destructive measurement of stress-free temperature in continuous welded rail", WCRR99, DS3-3, 1999.

[9] Vortok International : An operator's guide to VERSE - A non-destrauctive method of stress free temperature (SFT) measurement, United Kingdom Version, 2000.

[10] T. Livingston, J.G. Béliveau, D.R. Huston, "Estimation of axial load in prismatic members using flexural vibrations", Journal of Sound and Vibration, 179(5), 899-908, 1995.

[11] Y. Luo, L. Li, H. Yin, "A dynamic analysis of a continuous welded rail track under a longitudinal stress caused by temperature changes", Proceedings of IMechE F: Journal of Rail and Rapid Transit, 224(2), 91-101, 2010.

[12] R. Weaver, "Vibration measurement of rail stress", IDEA Program Final Report, 2006.

[13] V. Damljanović, R.L. Weaver, "Laser vibrometry technique for measurement of contained stress in railroad rail", Journal of Sound and Vibration, 282, 341-366, 2005. 
[14] G. Kjell, E. Johnson, "Measuring axial forces in rail by forced vibrations: experiences from a full-scale laboratory experiment", Proceedings of IMechE F: Journal of Rail and Rapid Transit, 223, 241-254, 2009.

[15] S. Shimizu, K. Abe, A. Aikawa, K. Koro, "Wave propagation analysis by 3D beam elements for an infinite track having axially loaded rails", Journal of Railway Mechanics, JSCE, 14, 75-82, 2010 (in Japanese).

[16] D. Ishikawa, K. Abe, K. Koro, "Dynamic analysis of an axially loaded curved rail", JASCOME, 10, 57-62, 2010 (in Japanese).

[17] K. Abe, S. Shimizu, A. Aikawa, K. Koro, "Theoretical study on a measuring method of rail axial stress via vibration modes of periodic track", WCRR2011, USB, 2011.

[18] T. Akutsu, K. Abe, D. Ishikawa, K. Koro, "Dynamic response analysis of continuous welded rails having a railroad switch subjected to a thermal stress", JASCOME, 11, 41-46, 2011 (in Japanese).

[19] T. Akutsu, K. Abe, K. Koro, "Influence of mechanical nonlinearity in railway track on resonant frequency-rail axial load relationship", Journal of Railway Mechanics, JSCE, 17, 105-112, 2013 (in Japanese).

[20] A. Aikawa, H. Sakai, K. Abe, "Numerical and experimental study on measuring method of rail axial stress of continuous welded rails based on use of resonant frequency", QR of RTRI, 54(2), 118-125, 2013.

[21] M. Suzuki, D. Sato, S. Mamada, S. Tamagawa, T. Deshimaru, "Improvement of the low-temperature characteristics of resilient rail pads by foam structuring", RTRI Report, 28(2), 17-22, 2014 (in Japanese).

[22] E. Tassilly, "Propagation of bending waves in a periodic beam", International Journal of Engineering Science, 25, 85-94, 1987.

[23] F. Urakawa, "Simple measuring method of the change in the CWR axial force based on the natural frequency", Journal of Railway Engineering, JSCE, 21, 8390, 2017 (in Japanese). 